\title{
Econograma: Diseño de un Instrumento Cualitativo para Investigación en Ambientes Sociales con poca o nula Información Socioeconómica de base*
}

\section{Econogram: designing a tool for qualitative research in social contexts with low or no socioeconomic information data base.}

Recibido: 12 de agosto de 2014 | Revisado: 1 de febrero de 2015 | Aceptado: 19 de marzo de 2015

doi:10.11144/Javeriana.upsy14-2.edic

Para citar este artículo: Páez, F. J., Bateman, A., Núñez, J., Hurtado-Caycedo, C., Gutiérrez, C. M., \& Pinzón, C. (2015). Econograma: diseño de un instrumento cualitativo para investigación en ambientes sociales con poca o nula información socioeconómica de base. Universitas Psychologica, 14(2), 599-604. http:// dx.doi.org.10.11144/Javeri-ana.upsy14-2.edic

* Artículo de investigación científica.

** Psicólogo, Máster en Psicología de las Organizaciones, del Trabajo y de los Recursos Humanos, y Magíster en Ciencias Sociales y Humana, Universidad de París V (Francia). Docente de la Pontificia Universidad Javeriana, Católica y Escuela de Administración de Negocios (EAN). franciscojavier22@gmail.com

**** Antropólogo de la Universidad de los Andes con Maestría en Historia de la Universitat Pompeu Fabra de Barcelona, España. Actualmente, está realizando una maestría en Global Studies en la Humboldt Universität zu Berlin de Berlín, Alemania. andres. bateman@gmail.com

***** Ingeniero Civil con maestría en Economía y Candidato a $\mathrm{PhD}$ en Ciencias Sociales y Humanas. Actualmente es investigador de Fedesarrollo, asesora al Gobierno nacional en los temas sociales y es profesor de la Universidad Javeriana. jairo.jnunez@gmail.com

******** Psicóloga. Máster en Estudios Culturales. Actualmente trabaja como asistente de investigación en Fedesarrollo.claudiahurtadoc@gmail.com

******* Administrador de empresas. Actualmente se desempeña como asistente de investigación en el centro de investigaciones económicas y sociales, Fedesarrollo.carlosgumar@gmail.com

*********** Socióloga de la Universidad Nacional de Colombia y Magíster en Estudios Culturales de la Universidad de los Andes. Actualmente se encuentra trabajando como asistente de investigación en Fedesarrollo. cpinzon@fedesarrollo.org.co

\author{
Francisco Javier Páez Becerra ** \\ Pontificia Universidad Javeriana, Bogotá, Colombia \\ ANDRÉS BATEMAN *** \\ JAIRO NÚÑEZ **** \\ Claudia Hurtado-CAYCEDO ****** \\ CARLOS MARIO GUTIÉRREZ ****** \\ CAROL Pinzón MÁSMEla ******** \\ Fundación para la Educación Superior y el Desarrollo, Bogotá, Colombia
}

RESUMEN

Este artículo presenta un instrumento de investigación cualitativa denominado econograma y tiene su origen en la necesidad generar un conocimiento de base para evaluar la eficacia de los programas estatales implementados en la atención de población damnificada por un fenómeno de excesiva pluviosidad, sucedido en Colombia entre noviembre de 2010 y marzo de 2011. Esta herramienta de investigación es a la vez un formato y una técnica, que pone en evidencia la estructura básica de las interacciones que se establecen entre un individuo o un colectivo en términos económicos, y permite rastrear desplazamientos en los comportamientos económicos a través del tiempo, desde una perspectiva que permite inferir ciclos de producción y de consumo, tendencias y redes de producción.

Palabras clave

investigación cualitativa; instrumentos de investigación; investigación social; econometría; línea de base; metodología de investigación

\begin{abstract}
A B S T R A C T
This paper presents a qualitative research tool called Econogram. This instrument has its origin in the need to generate new knowledge to evaluate the effectiveness of the state programs, implemented to attend the population affected by a phenomenon of excessive rainfall in Colombia between November 2010 and March 2011. This research tool is both a format and a technique, which proves the structure of the interactions between an individual, or a group in economic terms. It also allows tracking and understanding shifts in economic behavior throughout time to infer cycles of production, consumption and production networks and trends.

Keywords

qualitative research; research tools; social research; econometrics; baseline; research methodology
\end{abstract}




\section{Introducción}

Esta herramienta de investigación en ciencias sociales tiene su origen en una batería de instrumentos que fueron diseñados para desarrollar un proceso de evaluación de los programas que el Estado colombiano implementó para atender a la población damnificada por el fenómeno de La Niña 2010. 2011. Se consideraron las técnicas etnográficas de uso generalizado como la observación directa participante y no participante, los grupos focales y las entrevistas, para ahondar en la experiencia de la población damnificada y poder evaluar la eficacia de la atención del Estado. Sin embargo, se detectó una dificultad importante por no tener información oficial de la actividad económica y del tipo y calidad de las viviendas de las comunidades en los momentos previos al fenómeno meteorológico.

Los programas que generó el Estado buscaban, en primera medida, atender la emergencia derivada del fenómeno de la Niña 2010-2011, ofreciendo soluciones de vivienda temporal para preservar la vida de las familias damnificadas y, en segunda instancia, reactivar la economía en las regiones más golpeadas por la excesiva pluviosidad.

Si bien, el fenómeno de la Niña 2010-2011 tuvo incidencia en todo el territorio nacional, los peores desastres se localizaron en las cuencas hidrográficas de mayor caudal y que tradicionalmente se caracterizan por el difícil acceso, razón por la cual no se tenía actualizada la información sociodemográfica y económica en las zonas de mayor afectación.

La información inexistente, parcialmente inexistente o dispersa en diferentes fuentes oficia-

1 El ciclo conocido como El Niño, La Niña - Oscilación del Sur ENOS, es la causa de la mayor señal de variabilidad climática en la franja tropical del océano Pacifico, en la escala interanual. El Niño y su fase opuesta La Niña, son las componentes oceánicas del ENOS y corresponden, en términos generales, a la aparición, de tiempo en tiempo, de aguas superficiales relativamente más cálidas (El Niño) o más frías (La Niña) que lo normal en el Pacífico tropical central y oriental frente a las costas del norte de Perú, Ecuador y sur de Colombia. Estas alteraciones de la estructura térmica superficial y subsuperficial del océano están asociadas con el debilitamiento de los vientos alisios del Este y con el desplazamiento del núcleo de convección profunda del Oeste al Centro del Océano Pacífico tropical, en condiciones El Niño o con su permanencia e intensificación en el caso de La Niña. les y no oficiales no permitía establecer una línea de base que sirviera como referente tanto de la magnitud de la afectación que tuvo el fenómeno meteorológico en las personas y sus actividades productivas como en la efectividad de los programas de atención para la población damnificada que implementó el Estado.

En este escenario, una evaluación de los programas estatales demandaba un diseño metodológico que permitiera suplir la falta de información de base. Por ello, como lo señalan Mertens (2005) y Coleman y Unrau (2005) la metodología cualitativa resultó pertinente, en cuanto a que no se tenían referentes cuantitativos previos al fenómeno social que se deseaba caracterizar y debido a que la complejidad del fenómeno requería un abordaje preliminar de índole comprensivo.

\section{Insuficiencia de otros instrumentos cualitativos para la investigación socioeconómica en ambientes con poca o nula información de base}

El proceso de evaluación de los programas estatales se realizó al cabo del primer año de intervención y, por tanto, utilizó como insumo alguna información global que se obtuvo a lo largo de la atención a la población afectada por el fenómeno de La Niña. La información más precisa tuvo que ser levantada en los ambientes naturales de los participantes de los programas de reactivación económica y soluciones temporales de vivienda bajo las modalidades de albergues temporales, reparación de vivienda y de subsidios de arrendamiento para las familias damnificadas.

La impronta cualitativa de esta evaluación se evidencia en que nunca tuvo por pretensión la manipulación ni el control de variables. Como dirían Rothery, Tutty y Grinnell (1996) nunca "se buscó la reducción de los datos a valores numéricos". Por el contrario, a la luz del esquema de cadena de valor, se trató de comprender los significados construidos por la población participante, respecto de cada uno de los programas estatales desde su diseño, insumos y operación hasta el impacto que produjeron y su sostenibilidad. 
Inicialmente, se consideró el uso de la cartografía social ${ }^{2}$ como un instrumento para acceder a los significados que tenían los participantes respecto a su territorio y las afectaciones que trajo el fenómeno de La Niña 2010-2011. Como lo señalan Boada y Salazar (2005) son notables las bondades de la cartografía social y es recomendable su uso para entrar en el campo simbólico de los grupos humanos, sin embargo, dados los requerimientos de la evaluación de los programas estatales, se observó que, si bien daba cuenta de las actividades productivas, no permitía jerarquizar las actividades productivas según los niveles de importancia para los participantes y dar cuenta de la afectación del fenómeno meteorológico en ellas. Tampoco permitía incluir referentes cuantitativos de esa actividad productiva y, adicionalmente, resultaría muy dispendioso la realización de dos cartografías, para dar cuenta de los momentos pre y post fenómeno de La Niña 2010-2011.

\section{Econograma: herramienta para la estimación de una línea de base en perspectiva cualitativa}

La herramienta de investigación cualitativa que se generó para atender los requerimientos de la evaluación de los programas del Estado en las condiciones de emergencia humanitaria se ha denominado econograma. Con este nombre se ha querido enfatizar que este instrumento permite representar gráficamente la percepción que una población determinada tiene sobre un fenómeno socioeconómico dado y la incidencia que tienen otros factores sobre este.

Del mismo modo en que el sociograma resulta ser un método adecuado para conocer la estructura básica interrelacional de un grupo a través de las respuestas de sus componentes sobre sus propias atracciones y rechazos (Hernández, Fernández, \&

2 La Cartografía Social se funda en el concepto de "campo relacional"; concibe el territorio como referente espacio-temporal de la vida colectiva y hace de la construcción colectiva de mapas su herramienta para representarlos. En este sentido, "es un camino para el reconocimiento del mundo cultural, ecológico, productivo y político que se expresa en el territorio" (García, 2005).
Batista, 2012), el econograma pone en evidencia la estructura básica de las interacciones que se establecen entre un individuo o un colectivo en términos económicos. Esta metodología permite rastrear información a nivel diacrónico (desplazamientos de los comportamientos económicos a través del tiempo) y desde una perspectiva sistémica en los niveles micro, meso y macro sistémicos para inferir ciclos de producción y de consumo, tendencias y redes de producción.

El econograma fue diseñado atendiendo a seis condiciones propias de la investigación cualitativa: (1) expansividad, para permitir que diferentes conceptos relevantes puedan integrarse conforme se va desarrollando el estudio; (2) no directividad, en la medida en que está abierto a la construcción colectiva con los participantes del estudio; (3) experiencial, porque se fundamenta en la experiencia, percepción e intuición de los participantes más que en su explicación racional de un fenómeno; (4) flexibilidad, porque se adapta a un participante o a un grupo de informantes; (5) vivencial, porque permite consignar experiencias particulares, estimar procesos e incluso generar teorías fundamentadas en los diferentes puntos de vista de los participantes y (6) amplitud, en cuanto facilita la comprensión de las diferentes dimensiones internas, externas, pasadas y presentes de un fenómeno, ya que, como señala Creswell (2009), es fundamental que todo instrumento en investigación cualitativa permita situar el fenómeno de estudio en un contexto o ambiente para evitar que los resultados queden en un nivel general e inconexo de las experiencias cotidianas del grupo humano de referencia.

El econograma es el formato y la técnica de aplicación. Es el formato previo con que se inicia la conversación con los participantes de un estudio, así como la información que ellos consignan, los significados, las relaciones que establecen entre los elementos y las explicaciones que dan sobre el fenómeno abordado. Como se representa en la Figura 1, el formato del econograma consiste en círculos concéntricos que representan tanto las categorías y niveles de análisis como la jerarquía que se da entre ellas. Los círculos concéntricos están atravesados por una línea o eje vertical que 


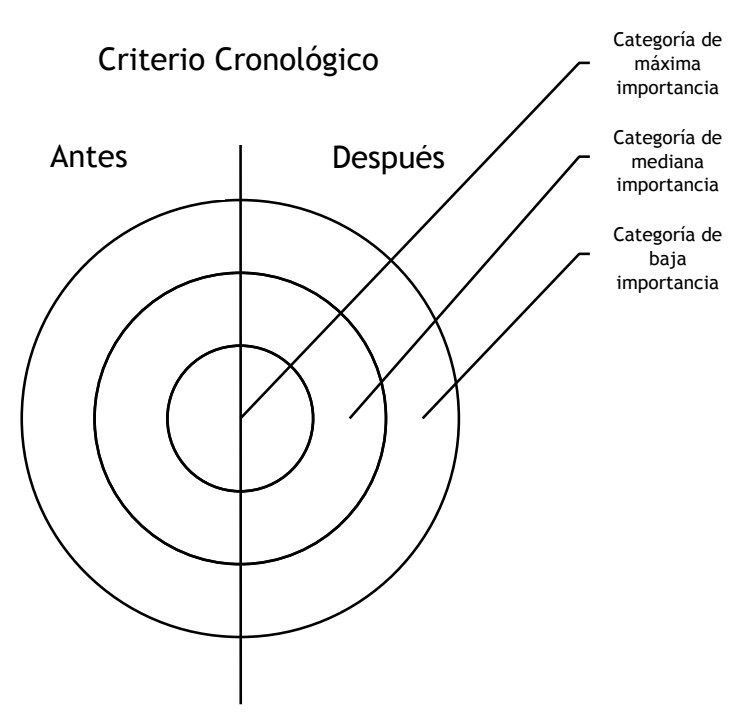

Figura 1. Formato del econograma.

Fuente: Elaboración propia.

permite representar un vector cronológico respecto del cual se sitúan las transiciones entre las fase previa y posterior al fenómeno.
La sencillez del formato da paso a la aplicación de la técnica del econograma. Como técnica cumple con dos criterios que Mertens (2005) denominó como accesibilidad y conveniencia. Accesibilidad, porque facilita la comprensión por parte de los participantes indistintamente de los diferentes niveles de escolaridad, género, edad, oficio, creencia o cualquier otra condición particular. De esta manera, todos los participantes se constituyen en informantes válidos o gatekeepers. El econograma cumple con el criterio de conveniencia porque el esquema básico tiene aplicación en diferentes contextos en los que se requiera una caracterización de las modulaciones en las actividades económicas a lo largo de la historia, desde la perspectiva tanto de individuos como grupos cuando no se tiene una línea de base o cuando se quiere generar una metodología mixta. El econograma puede ser aplicado a individuos, a casos-tipo o a muestras dirigidas. Sin embargo, es fundamental que se acote el alcance de los estudios cualitativos porque su pretensión no es la extrapolación de los resultados a la población general.

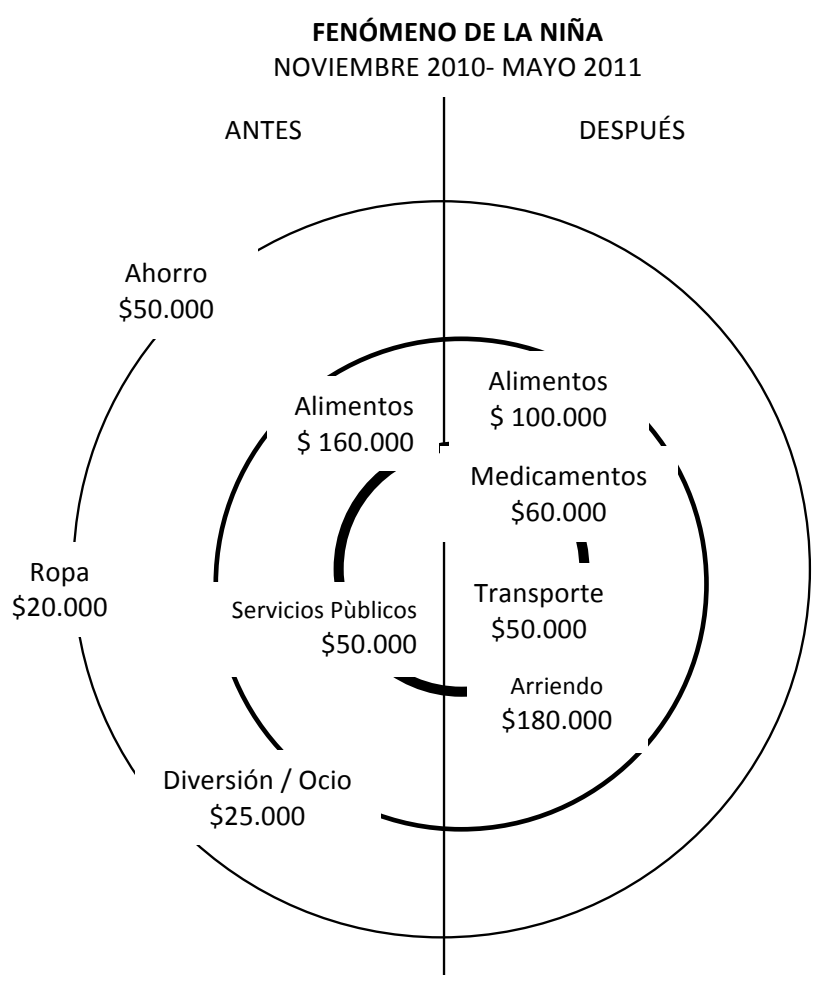

Figura 2. Caso de damnificado por el fenómeno de La Niña 2010-2011.

Fuente: Adaptado de casos frecuentes encontrados en el trabajo de campo en el marco del estudio de Nuñez, Hurtado-Caycedo, Páez, Pinzón, Gutiérrez y Castillo (2013). 
En el proceso de construcción, el econograma como formato y como técnica, fue validado por expertos en econometría e investigación cualitativa así como por especialistas en las áreas en las que se desarrolló la evaluación de los programas de reactivación económica y soluciones temporales de vivienda, reparación de vivienda y subsidio de arrendamiento. Especialistas y expertos coincidieron en dar una opinión favorable sobre este instrumento, que adicional a los criterios de accesibilidad y conveniencia, resultó ser muy sensible para que los participantes pudieran asignar un nivel jerárquico a la información y poner en evidencia las diferencias entre las actividades económicas previas y posteriores al fenómeno de La Niña 2010-2011, de manera que la investigación no solamente registraba hechos presentes. Y ciñéndose a lo que recomienda Williams, Unrau y Grinell (2005), el instrumento diseñado para la investigación cualitativa entiende a los participantes y sus contextos a través de la historia reciente. Uno de los especialistas en los programas estatales, reconoció la pertinencia del econograma para identificar a los líderes de opinión en el grupo de participantes, o lo que Willig (2008) denominó: informantes clave.

Los resultados obtenidos a través del econograma pueden ser procesados desde perspectivas inherentes al análisis de contenido y análisis de discurso, e incluso, pueden alcanzar un nivel de procesamiento desde la Teoría Fundamentada para establecer convergencias, divergencias o tendencias en las versiones que ofrecen los diferentes participantes de la investigación.

\section{Aplicación y aciertos del econograma como herramienta de investigación cualitativa}

La flexibilidad del instrumento facilita el acceso a una gran cantidad de información de orden socioeconómico. A continuación, en la Figura 2, para el caso de los damnificados por el fenómeno de La Niña 2010-2011, se observa cómo uno de los participantes pudo dar cuenta de su actividad económica previa al fenómeno meteorológico y cómo se alteró su actividad productiva.
En el caso de este participante cabeza de familia, se observa que, previo al fenómeno de La Niña 2010-2011, tenía una actividad económica fundamentalmente agrícola que desarrollaba en un predio de su propiedad en la región cercana al medio Atrato (Chocó), según lo reportó verbalmente. Esta actividad le aseguraba ingresos mensuales aproximados de $\$ 305.000$ pesos distribuidos como se observa en la Figura 2. Los rubros estaban dispersos en diferentes niveles de importancia, siendo los más importantes los referidos a la alimentación, la salud y el pago de servicios públicos. En segundo nivel de importancia, aparecían gastos referidos a la diversión/ocio, los gastos de ropa y el índice de ahorro que aparecían en el nivel más bajo de importancia.

Posteriormente a la temporada invernal causada por el fenómeno de La Niña 2010-2011, los compromisos económicos reportados por el participante llegan a $\$ 390.000$. A causa de la temporada invernal, el participante perdió los cultivos y el ganado menor que tenía en el predio, así como su domicilio pues quedó inhabitable. En estas condiciones, se ve obligado a asumir el pago de un arriendo, ha disminuido la inversión en alimentos, aumentó el gasto por medicamentos para atender los requerimientos de uno de los miembros de la familia del informante que padece una enfermedad crónica que empeoró por las condiciones húmedas del ambiente. El participante reportó que aumentó el gasto de transporte para que los hijos de la familia puedan asistir a la escuela, donde, además de la educación, reciben una ración de alimento. Como se observa, el rubro del ahorro desapareció como consecuencia de su condición de damnificado así como el dinero dedicado a la diversión u ocio. Todos los rubros aparecen clasificados en el nivel más alto de importancia y el participante reportó que están ahí porque eso es "lo mínimo para no dejarse morir". Afirma que organizaciones de ayuda humanitaria le colaboraron con el trasteo de los enseres básicos de la familia, pero que el resto de los muebles se perdieron por la inundación.

El econograma permitió, para este caso en particular, adquirir un punto de vista "interno" (Williams et al., 2005) del participante, respecto a cuestiones que se vinculan con el fenómeno que 
se estudia, lo cual facilita y fundamenta el análisis que hace el investigador como observador externo. En el desarrollo del econograma, se pudo acceder a conceptos, lenguaje y expresiones propias del participante.

Anastas (2005) valora la capacidad que tiene un instrumento de investigación para "enfocar" la atención de los investigadores en medio de mucha información que se presenta como dispersa e incluso desordenada. Este instrumento de investigación cualitativa facilitó que los investigadores concentraran su atención en aspectos relevantes para comprender el fenómeno y para alcanzar una mejor comprensión a través del uso de los demás instrumentos de investigación que se proyectaron para la evaluación de los programas de atención a los damnificados por la temporada invernal.

El instrumento permitió identificar procesos sociales fundamentales en el ambiente y determinar cómo opera, para este caso, el desplazamiento forzado por la situación de calamidad, las nuevas demandas de alimento, salud y vivienda que debe satisfacer y la pertinencia y suficiencia de los programas que creó el Estado para atender la emergencia humanitaria.

El econograma permitió describir y comprender la configuración de los roles sociales en un grupo determinado y las alteraciones que se producen a lo largo del tiempo. Asimismo, facilitó la comprensión que alcanzan los participantes sobre la experiencia que han tenido sobre un fenómeno determinado.

Otras adaptaciones de este instrumento permitirían también identificar redes de producción antes y después de la incidencia de un fenómeno en un individuo o en un grupo. Igualmente, se podrían hacer aplicaciones colectivas de manera que se reflejen las interacciones sociales que se dan en razón a la actividad económica.

\section{Referencias}

Anastas, J. W. (2005). Observation. En R. M. Grinnell \& Y. A. Unrau (Eds.), Social work: Research and evaluation. Quantitative and qualitative approaches (7.a ed., pp. 213-230). New York: Oxford University Press.

Boada, A., \& Salazar, D. (2005). Producción de la cartografía para el censo general de 2005: aporte a la infraestructura colombiana de datos espaciales. Análisis geográficos. Revista del Instituto Geográfico Agustín Codazzi, 29, 7-12.

Coleman, H., \& Unrau, Y. A. (2005). En R. M. Grinnell \& Y. A. Unrau (Eds.), Social work: Research and evaluation. Quantitative and qualitative approaches (7.a ed., pp. 403-420). New York: Oxford University Press.

Creswell, J. W. (2009). Research Design: qualitative, quantitative, and mixed methods approaches (3. . $^{-}$ ed.). Londres: Sage.

García, C. (2005). Barrios del mundo: historias urbanas. La cartografía social en la práctica. Bogotá: ENDA Colombia. Disponible en http://www.quartiersdumonde.org/uploads/documento/archivo/25/Documento_de_sistematizacion_de_la_utilizacion_de la_Cartografia_social_durante_el_primer_ano_ del_proyecto.pdf

Hernández, R., Fernández, C., \& Baptista, M. (2010). Metodología de la investigación. México: McGraw Hill.

Mertens, D. (2005). Research and evaluation in education and psychology. Integrating diversity with quantitative, qualitative, and mixed methods (2.a ed.). Thousand Oaks, CA: Sage.

Nuñez, J., Hurtado-Caycedo, C., Páez, FJ., Pinzón, C., Gutiérrez, C, y Castillo J. (2013) Evaluación de los programas para la atención del fenómeno de la Niña 2010/2011. Colombia: La imprenta editores.

Rothery, M., Tutty, L., \& Grinell, R. (Eds.). (1996). Qualitative research for social workers: Phases, steps and tasks. Boston: Allyn \& Bacon.

Williams, M., Grinnell, R. M., \& Unrau, Y. A. (2005). Case levels design. En R. M. Grinnell \& Y. A. Unrau (Eds.), Social work: Research and evaluation. Quantitative and qualitative approaches (7.a ed., pp. 171-184). New York: Oxford University Press.

Willig, C. (2008). Introducing qualitative research in psychology: Adventures in theory and method. Philadelphia: Open University Press. 